\title{
USO DA TÉCNICA HRICP-MS NA AVALIAÇÃO DOS ISÓTOPOS DE BORO EM EUCALIPTO
}

\author{
Edson Marcio Mattiello*, Hugo Alberto Ruiz e Ivo Ribeiro da Silva \\ Departamento de Solos, Universidade Federal de Viçosa, 36570-000 Viçosa - MG, Brasil \\ Jorge Eduardo de Souza Sarkis \\ Instituto de Pesquisas Energética e Nucleares, 05580-000 São Paulo - SP, Brasil
}

Recebido em 26/2/10; aceito em 21/9/10; publicado na web em 7/1/11

\begin{abstract}
USE OF THE HRICP-MS TECHNIQUE FOR THE EVALUATION OF BORON ISOTOPES IN EUCALITPUS PLANTS. The ${ }^{10} \mathrm{~B}$ isotope tracer technique is essential to study the B mobility in plants. Factors that can influence the quality of measured B isotope ratios were optimized experimentally using High Resolution Inductively Coupled Plasma Mass Spectrometry (HRICP-MS). An isotopically certified standard (NIST SRM-951) was used. The best combination was obtained using a resolution of 400, a RF power of $1250 \mathrm{~W}$, followed by 15 measurements over a 10-s integration period each (15*10). Utilizing this approach it was possible to obtain a precision of $0.3 \%$ in standard material and $2 \mathrm{z} \%$ in the experimental samples. The results show the importance of establishing optimized work conditions before carrying out the analytical series.
\end{abstract}

Keywords: isotope ratio; micronutrients; translocation.

\section{INTRODUÇÃO}

O boro (B) é um elemento quimicamente complexo; depois do carbono, deve ser o elemento de característica físico-química mais intrigante. ${ }^{1}$ É um elemento essencial para as plantas e sua deficiência afeta o crescimento e produção das culturas em diversas regiões do mundo. ${ }^{2}$ No solo, os teores disponíveis para as plantas variam entre 0,1 e $2 \mathrm{mg}$ $\mathrm{dm}^{-3}$ de B (B extraído pelo método água quente), ${ }^{3} \mathrm{e}$ teores foliares entre 20 e $100 \mathrm{mg} \mathrm{kg}^{-1}$ são considerados adequados para a maioria das plantas cultivadas. ${ }^{4}$ Os teores de $\mathrm{B}$ encontrados em tecido vegetal têm sido usados como indicativo do estado nutricional desse elemento nas plantas.

Diversas técnicas têm sido usadas para determinação de B no solo e em tecido vegetal. ${ }^{5} \mathrm{O}$ método colorimétrico é o mais difundido e baseia-se na formação de complexo colorido com acido bórico a pH 5,1, utilizando azometina-H. A intensidade da cor é medida a 420 nm, em espectrofotômetro ou colorímetro. É um método simples, de rápida execução e com sensibilidade apropriada para a maioria dos estudos de fertilidade do solo e nutrição de plantas. A determinação de elementos-traços, entre eles o B, tem sido facilitada após a introdução dos espectrofotômetros de emissão óptica com plasma indutivamente acoplado (ICP-OES), sobretudo pelo baixo limite de detecção, pela ampla faixa de linearidade e pela determinação múltipla de elementos. ${ }^{6}$ No entanto, a determinação do teor total de B em tecido vegetal não permite o conhecimento da redistribuição ou remobilização do nutriente entre os órgãos da planta. Também não permite distinguir a origem do B nos estudos de fertilização e absorção do nutriente pelas plantas. Assim, estudos da razão isotópica de B em amostras vegetais são de fundamental importância para a compreensão da absorção e do transporte do nutriente internamente na planta, sendo relevante na distinção dos genótipos quanto à aquisição e utilização do nutriente ${ }^{7}$ e na orientação de técnicas mais apropriadas de fertilização.

As técnicas tradicionalmente empregadas na determinação de razões isotópicas de B em amostras vegetais são a espectrometria de massas termoiônica (TIMS) e a espectrometria de massas de íon secundário

\footnotetext{
*e-mail: mattiello@ufv.br
}

(SIMS) ${ }^{8,9}$ A TIMS é a técnica mais precisa e sensível e permite a análise por meio de íons positivos ${ }^{10}$ ou negativos. ${ }^{11} \mathrm{~A}$ grande desvantagem desta técnica se deve a interferências isobáricas, exigindo a separação química do elemento de interesse. A técnica SIMS possui a vantagem de permitir a determinação simultânea da concentração e composição isotópica do B nas amostras em estudo sem nenhum tratamento prévio, porém a precisão dos resultados é inferior à da técnica anterior (cerca de 3-4\%), o que torna difícil a observação de pequenas variações na intensidade isotópica a ser medida.

A partir de meados da década de 1970 surgiu uma nova instrumentação analítica, resultante do acoplamento de uma fonte de íon extremamente eficiente (plasma indutivamente acoplado-ICP) com uma técnica de análise iônica extremamente seletiva (espectrometria de massas-MS). Essa técnica, denominada espectrometria de massas com fonte de plasma indutivamente acoplado (ICP-MS), rapidamente se popularizou e tem sido utilizada nas mais diversas áreas do conhecimento. ${ }^{12-14}$ Atualmente, duas categorias de equipamentos dessa natureza estão disponíveis: os chamados de baixa resolução, cuja separação iônica é feita, fundamentalmente, por filtros quadrupolos; e aqueles conhecidos como de alta resolução ou setor magnético, nos quais a análise do feixe iônico é realizada por meio de um sistema conhecido como Nier-Johnson invertido, no qual um analisador magnético está acoplado a um setor elétrico. ${ }^{15}$

Ambas as técnicas têm sido utilizadas nos estudos de isótopos de B. ${ }^{16}$ Técnicas de determinação dos dois isótopos estáveis de $\mathrm{B},{ }^{10} \mathrm{~B} \mathrm{e}{ }^{11} \mathrm{~B}$, com abundância natural aproximada de 19,9 e 80,1\%, respectivamente, permitem o monitoramento do B em sistemas biológicos. O baixo limite de detecção é uma grande vantagem em relação aos demais métodos, podendo variar de 1 a $3 \mu \mathrm{g} \mathrm{L}^{-1}$ de B em extratos biológicos digeridos. ${ }^{17,18}$ A determinação de $\mathrm{B}$ por meio da diluição isotópica em ICP-MS é considerada a mais exata técnica para análises quantitativas. ${ }^{19}$

Análises isotópicas de B normalmente não sofrem interferências espectroscópicas. Entretanto, vários outros fatores podem influenciar a qualidade dos resultados analíticos, entre os quais: tempo morto dos detectores, efeito espaço/carga, eficiência de transmissão iônica, estabilidade da corrente iônica e resolução espectral. ${ }^{12}$ Todos esses 
parâmetros podem ser otimizados experimentalmente, por meio da análise de material de referência certificado isotopicamente; contudo, são poucos os relatos na literatura sobre as condições analíticas adequadas para análise da razão isotópica do B em material vegetal, por meio de espectrometria de massas de alta resolução com fonte de plasma ativado indutivamente (HRICP-MS).

A utilização de técnicas sensíveis e capazes de determinação de B com alta precisão e exatidão é fundamental para avaliações de razões isotópicas de $\mathrm{B}$ nos estudos de fertilização e absorção de $\mathrm{B}^{20} \mathrm{e}$ de mobilidade do nutriente em plantas. ${ }^{7,21}$ Neste trabalho, objetivou-se avaliar a técnica HRICP-MS na determinação da razão isotópica de B em amostras de tecido de plantas de eucalipto submetidas à aplicação de ${ }^{10} \mathrm{~B}$ e sua aplicação para estudos de mobilidade de B em plantas. Para isso, foram avaliados os principais fatores que influenciam a qualidade das medidas da razão isotópica do B, como a estatística de contagem, a potência da radiofrequência, a qualidade do espectro e o ajuste do sistema óptico do aparelho.

\section{PARTE EXPERIMENTAL}

\section{Reagentes e soluções}

Todos os reagentes utilizados apresentavam pureza analítica. O ácido nítrico (Merck, Darmstadt, Germany) foi preparado com água com resistividade de $18,2 \mathrm{M} \Omega \mathrm{cm}^{-1}$, obtida em sistema MilliQ (Millipore, Bedford, MA, USA). A solução de B, enriquecida com ${ }^{10} \mathrm{~B}\left(99\right.$ atom $\left.\%{ }^{10} \mathrm{~B}\right)$, foi preparada a partir de $\mathrm{H}_{3} \mathrm{BO}_{3}$ (Aldrich). A solução estoque de $1.000 \mathrm{mg} \mathrm{L}^{-1}$ de $\mathrm{B}$ foi preparada a partir de material de referência certificado NIST SRM-951, com 19,827\% de ${ }^{10} \mathrm{~B}$ e $80,173 \%$ de ${ }^{11} \mathrm{~B}$. Padrões contendo $91,85 \mu \mathrm{g} \mathrm{L}^{-1}$ de $\mathrm{B}$ foram preparados a partir da solução estoque padrão e usados na fase de calibração e durante as séries analíticas. A concentração do padrão era próxima das concentrações esperadas nos extratos diluídos das amostras a serem analisadas.

\section{Obtenção do material vegetal}

Mudas de dois clones comerciais de eucalipto, codificados sob os números 68 e 129, foram cultivadas em solução nutritiva, ${ }^{22} 1 / 2$ força, com $10 \mu \mathrm{mol} \mathrm{L}-1$ de $\mathrm{B}$ (abundância natural), sendo a fonte $\mathrm{H}_{3} \mathrm{BO}_{3}$ (Merck, Darmstadt, Germany), durante 21 dias, em casa de vegetação. Após esse período, selecionaram-se plantas uniformes de cada clone. Dois terços das plantas foram cultivados na ausência de B, e o terço restante, na presença de $20 \mu \mathrm{mol} \mathrm{L}^{-1}$ de B (abundância natural). Decorridos 45 dias nessas condições, uma única folha madura das plantas crescidas na ausência e de outras crescidas na presença de B foi imersa em solução de $\mathrm{H}_{3} \mathrm{BO}_{3}$ enriquecido com 99 atom $\%{ }^{10} \mathrm{~B}$, na concentração de $1,64 \mathrm{~g} \mathrm{~L}^{-1}$ de ${ }^{10} \mathrm{~B}$, durante $1 \mathrm{~min}$. Outras plantas cultivadas na ausência de $\mathrm{B}$ não receberam a solução de ${ }^{10} \mathrm{~B}$, permanecendo como testemunhas. Precauções foram tomadas para a não contaminação de outras partes da planta e da solucão nutritiva. Dessa forma, podia-se verificar qualitativa e quantitativamente a translocação de B da folha madura para outras partes da planta.

Nos tempos de 1, 5, 12 e 17 dias após a imersão da folha madura em solução contendo ${ }^{10} \mathrm{~B}$, foram retiradas amostras de tecidos jovens (folhas jovens e ápices dos ramos), dos três tratamentos impostos. Previamente à imersão da folha madura em solução contendo ${ }^{10} \mathrm{~B}$ foram retiradas amostras de tecidos jovens para referenciar o tempo zero.

\section{Preparo das amostras}

As amostras dos tecidos jovens foram secas em estufa de circulação forçada a $65^{\circ} \mathrm{C}$ por $96 \mathrm{~h}$. Elas foram trituradas e, aproximada- mente, $0,2 \mathrm{~g}$ do material foram calcinados a $550{ }^{\circ} \mathrm{C}$ em cadinhos de porcelana; em seguida, o resíduo foi dissolvido em $5 \mathrm{~mL}$ de ácido nítrico, na concentração de $1,59 \mathrm{~mol} \mathrm{~L}^{-1}$. O resíduo foi passado por filtro de náilon de $0,45 \mu \mathrm{m}$ e armazenado em microtubos plásticos em ultrafreezer.

\section{Procedimento analítico e obtenção dos dados}

Para a determinação da razão isotópica de ${ }^{10} \mathrm{~B}:{ }^{11} \mathrm{~B}$ utilizou-se um espectrômetro de massa de alta resolução com fonte de plasma ativado indutivamente (HRICP-MS, Element-Thermo Finnigan, Bremen, Germany), nas condições de operação apresentadas na Tabela 1.

Tabela 1. Condições de operação do HRICP-MS

\begin{tabular}{|c|c|}
\hline Instrument & Element ( Thermo Finnigan, Bremen, Germany) \\
\hline Sensitivity & $10 \mathrm{ng} \mathrm{g}^{-1115} \mathrm{In}=1 \times 10^{6}$ counts \\
\hline rf Power & $1250 \mathrm{~W}$ \\
\hline Nebulizer & $\begin{array}{c}\text { Meinhardt glass with water cooled spray chamber } \\
\text { Scott type }\end{array}$ \\
\hline Sample uptake & $0.8 \mathrm{~mL} \mathrm{~min}^{-1}$ \\
\hline Interface & Ni cones \\
\hline Gas flows $\left(\mathrm{L} \mathrm{min} \mathrm{min}^{-1}\right)$ & Plasma $=14$, auxiliary 1.0, nebulizer $=0.90$ \\
\hline Resolution $\mathrm{m} / \Delta \mathrm{m}$ & 300 , \\
\hline Integration window & $80 \%$ \\
\hline Scans & Eletric, number of scans $=30$ \\
\hline Detector & Ion counting \\
\hline
\end{tabular}

Soluções padrão NIST SRM-951, de razão ${ }^{10} \mathrm{~B} /{ }^{11} \mathrm{~B}$ equivalente a 0,247, foram analisadas em diferentes combinações de potência da radiofrequência, focus e método de contagem. Também foi avaliada a estabilidade da leitura ao longo do tempo. A melhor combinação, a que mais se aproximasse do padrão certificado NIST SRM-951 e com menores valores de coeficiente de variação (CV), foi considerada apropriada para realização das séries analíticas.

Uma vez estabelecidas as condições de trabalho mais adequadas para as análises (calibração), procedeu-se à determinação da razão isotópica de $\mathrm{B}\left({ }^{10} \mathrm{~B}:{ }^{11} \mathrm{~B}\right)$ em tecido de eucalipto. As amostras foram diluídas 10 vezes com água deionizada $18,2 \mathrm{M} \Omega \mathrm{cm}^{-1}$ e colocadas em amostrador automático, determinando-se a razão isotópica de ${ }^{10} \mathrm{~B}:{ }^{11} \mathrm{~B}$ nos extratos.

\section{RESULTADOS E DISCUSSÃO}

Inicialmente, foram estudados os principais fatores que poderiam influenciar a qualidade da razão isotópica: estatística de contagem, potência da radiofrequência, qualidade do espectro e ajuste do sistema óptico do aparelho. As amostras analisadas nessa fase (padrão certificado NIST 951) representaram diferentes combinações de fatores interferentes na razão isotópica de $\mathrm{B}$. Os resultados mostraram que a qualidade da determinação da razão isotópica (exatidão e precisão) depende de ajustes específicos do aparelho e do método adotado (Figura 1). A aproximação dos valores medidos no aparelho ao padrão NIST 951 foi possível quando se adotaram canais de 400 (resolução de massa, $\mathrm{M} / \Delta \mathrm{M}$ ).

A potência da radiofrequência foi a variável de maior influência na razão ${ }^{10} \mathrm{~B} /{ }^{11} \mathrm{~B}$, e o melhor ajuste, em relação ao padrão NIST 951, foi obtido utilizando valores de $1.250 \mathrm{~W}$ (Figura 1). A seleção de valores de resolução de massa mais elevados (3800) em HRICP-MS resultou em interferências do ion ${ }^{40} \mathrm{Ar}^{4+}$ na leitura do ${ }^{10} \mathrm{~B}$ devido à pequena diferença entre o número de massa de ambos. Essa interferência foi 

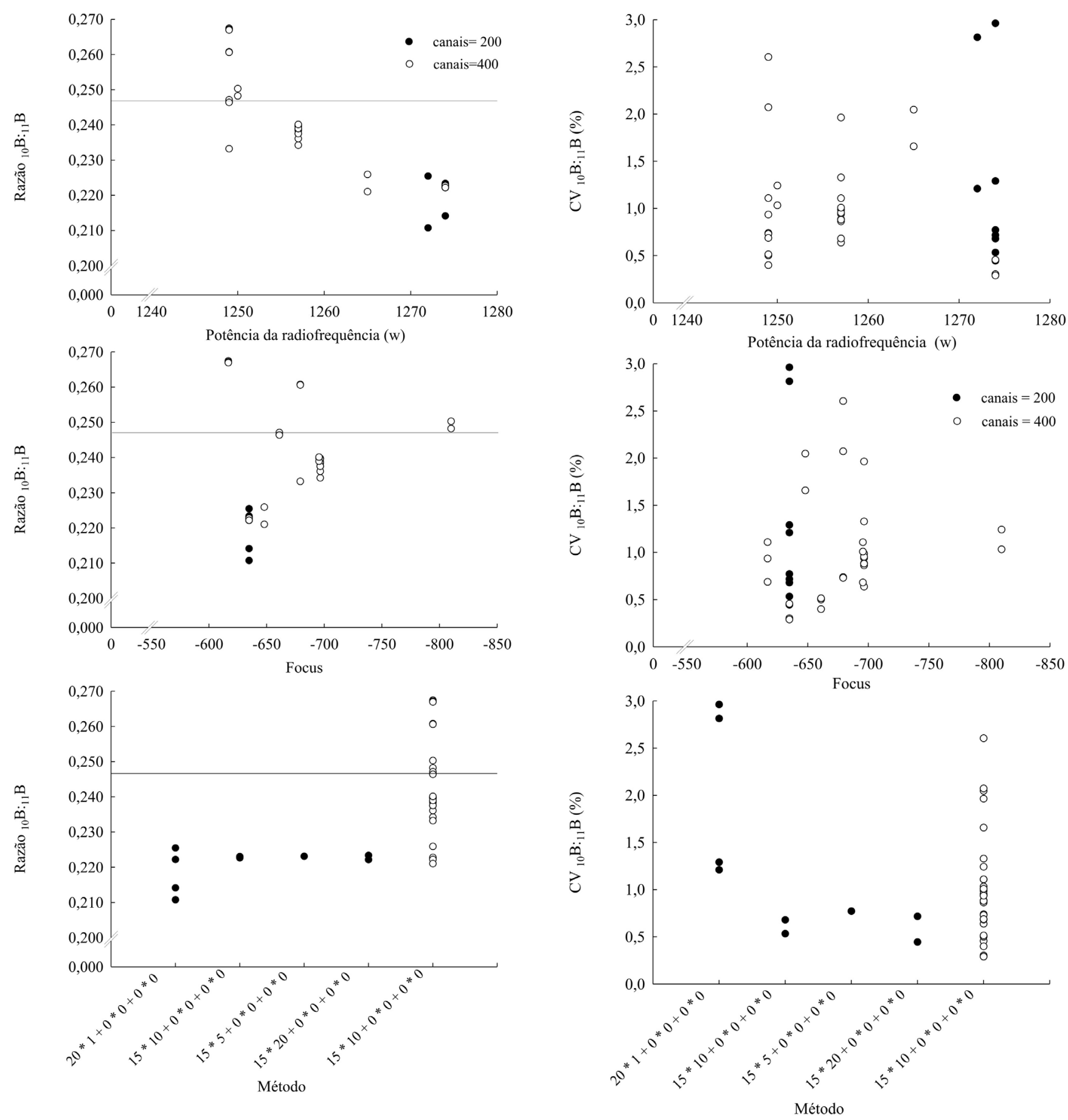

Figura 1. Razão isotópica de B considerando a potência da radiofrequência, focus e método, utilizando canais de 200 e 400. O traço (-) indica a razão isotópica do padrão NIST SMR 951

reduzida com a adoção de uma menor resolução (350) e menor valor de potência da radiofrequência $(1200 \mathrm{~W}) .{ }^{23}$ Operando em condições de menor resolução o sinal geralmente tem maior intensidade e os picos tem formato de topo achatado, resultando em maior precisão da medição da razão isotópica do B. ${ }^{23}$

$\mathrm{O}$ focus utilizado também influenciou a razão isotópica de B. Valores menores que -650 se mostraram mais apropriados para determinação da razão isotópica (Figura 1).

A calibração do aparelho em relação ao padrão NIST 951 foi possível apenas quando se adotou estatística de contagem de $15^{*} 10$ (15 medições com 10 períodos de integração) (Figura 1). As demais

Figura 2. Coeficiente de variação (CV\%) da razão isotópica de B, considerando a potência da radiofrequência, focus e método, utilizando canais de 200 e 400

combinações foram pouco exatas e precisas, sendo, portanto, descartadas nas determinações posteriores à fase de calibração.

O coeficiente de variação também foi influenciado pelas diferentes combinações na fase de calibração (Figura 2). Considerando como satisfatório nessa fase um CV menor que $1,5 \%$, percebe-se que uma ampla faixa de potência da radiofrequência atende a essa précondição. Foi possível obter CV menor que 0,3\% durante essa fase, evidenciando elevada precisão nas leituras do padrão. Em relação ao focus utilizado, foi possível obter CV menor que 1,5\% em uma ampla faixa de trabalho. 
Na Figura 3 é apresentada a razão ${ }^{10} \mathrm{~B} /{ }^{11} \mathrm{~B}$ em amostras de tecidos jovens de dois clones de eucalipto que receberam uma única aplicação de ${ }^{10} \mathrm{~B}$ numa folha madura. A técnica usada mostrou-se precisa e exata, possibilitando a detecção do enriquecimento de ${ }^{10} \mathrm{~B}$ nos órgãos jovens da planta, com destaque para o clone 129 , que se mostrou mais eficiente na translocação do B. Portanto, essa técnica, sob condições analíticas definidas, será extremamente valiosa em estudos futuros que envolvam a absorção ou redistribuição floemática de B em eucalipto, ${ }^{7}$ ou mesmo auxiliar no isolamento de possíveis complexos orgânicos de B no floema, conforme já relatado para outras espécies. ${ }^{24}$

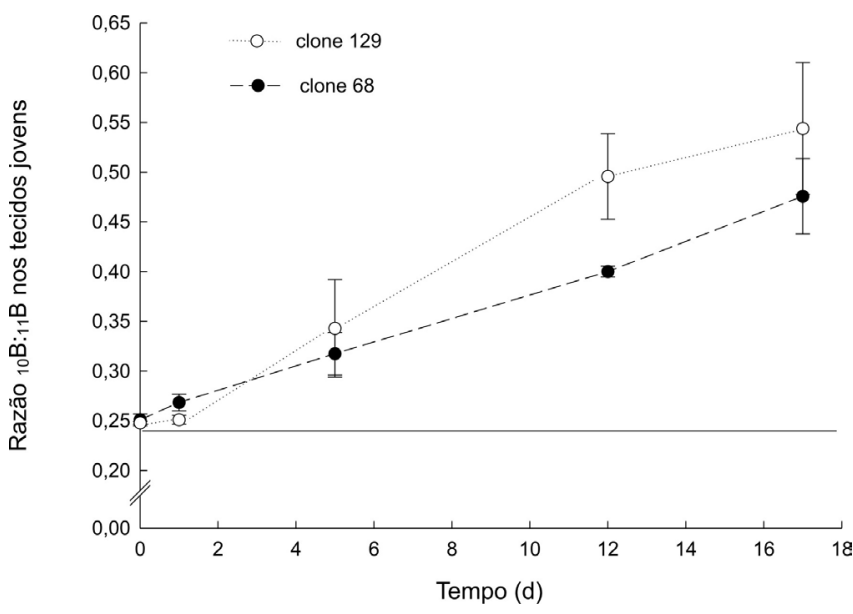

Figura 3. Razão isotópica de B em tecidos jovens de dois clones de eucalipto que receberam uma única aplicação de ${ }^{10} \mathrm{~B}$ na folha madura, considerando o tempo de aplicação de ${ }^{10} \mathrm{~B}$. O traço (-) indica a razão isotópica do padrão NIST SMR 951

\section{CONCLUSÕES}

Há necessidade do uso e correção por padrão certificado para correção das pequenas variações encontradas nas rotinas de trabalho.

Com o equipamento e as condições estabelecidas foi possível atingir coeficientes de variação de $0,3 \%$ para os padrões e de $2 \%$ para as amostras.

Por meio da determinação da razão isotópica de B em HRICPMS, foi possível verificar a mobilidade de B em plantas de eucalipto.

\section{MATERIAL SUPLEMENTAR}

Está disponível em http://quimicanova.sbq.org.br, na forma de arquivo PDF, com acesso livre. A razão isotópica de B do material de referência NIST SRM 951 foi ligeiramente afetada pelo tempo de duração da série analítica (Figura 1S). Os resultados mostraram va- riação da razão ${ }^{10} \mathrm{~B} /{ }^{11} \mathrm{~B}$ de 0,230 a 0,248 , sendo a média de 0,240 . Em média, a exatidão foi de $97,2 \%$ ao longo da série analítica. Os valores de razão ${ }^{10} \mathrm{~B} /{ }^{11} \mathrm{~B}$ para o material de referência no presente estudo são comparáveis com a faixa de $0,238-0,252$ reportado anteriormente. ${ }^{23}$

\section{AGRADECIMENTOS}

Ao Instituto de Pesquisas Energéticas e Nucleares-IPEN, pela realização das análises e ao Conselho Nacional de Desenvolvimento Científico e Tecnológico- CNPq, pelo apoio financeiro.

\section{REFERÊNCIAS}

1. Greenwood, N.; Earnshaw, A.; Chemistry of the Elements, $2^{\text {nd }}$ ed.; Butterworth-Heinemann: Oxford, 1997.

2. Shorrocks, V.; Plant Soil 1997, 193, 121.

3. Goldberg, S.; Shouse, P.; Lesch, S.; Grieve, C.; Poss, J.; Forster, H.; Suarez, D.; Soil Sci. 2002, 167, 720.

4. Malavolta, E.; Pimentel-Gomes, F.; Alcarde, J.; Adubos e adubações, $1^{\text {a }}$ ed.; Nobel: São Paulo, 2000.

5. Sah, R.; Brown, P.; Microchem. J. 1997, 56, 285.

6. Duffy, M.; Thomas, R.; Atom. Spectrosc. 1996, 17, 128.

7. Mattiello, E. M.; Ruiz, H. A.; Silva, I. R.; Sarkis, J. E. S; Neves, J. C. L.; Pucci, M. M.; R. Bras. Ci. Solo 2009, 33, 1695.

8. Derue, C.; Gibouin, D.; Verdus, M.; Lefebvre, F.; Demarty, M.; Ripoll, C.; Thellier, M.; Microsc. Res. Techniq. 2002, 58, 104.

9. Vering, G.; Crone, C.; Bijma, J.; Arlinghaus, H.; Appl. Surf. Sci. 2003, 203, 785 .

10. Deyhle, A.; Int. J. Mass Spectrom. 2001, 206, 79.

11. Shen, J.; You, C.; Anal. Chem. 2003, 75, 1972.

12. Heumann, K.; Gallus, S.; Radlinger, G.; Vogl, J.; J. Anal. Atom. Spectrom. 1998, 13, 1001.

13. Houk, R. S.; Accounts Chem. Res. 1994, 27, 333.

14. Jarvis, I.; Jarvis, K. E. Chem. Geol. 1992, 95, 1.

15. Vanhaecke, F.; Moens, L.; Dams, R.; Taylor, P.; Anal. Chem. 1996, 68, 567.

16. Brown, P.; Hu, H.; Ann. Bot. 1996, 77, 497.

17. Smith, F. G.; Wiederin, D. R.; Houk, R. S.; Anal. Chem. 1991, 63, 1458.

18. Evans, S.; Krahenbuhl, U.; J. Anal. At. Spectrom. 1994, 9, 1249.

19. Sah, R.; Brown, P.; Plant Soil 1997, 193, 15.

20. Franco, H.; Trivelin, P.; Vitti, A.; Otto, R.; Faroni, C.; Tovajar, J.; $R$. Bras. Ci. Solo 2009, 33, 1667.

21. Boaretto, R.; Quaggio, J.; Mourao, F.; Gine, M.; Boaretto, A.; Commun. Soil. Sci. Plan. 2008, 39, 2501.

22. Clark, R. B.; J. Agr. Food. Chem. 1975, 23, 458.

23. Gabler, H. E.; Bahr, A.; Chem. Geol. 1999, 156, 323.

24. Hu, H. N.; Penn, S. G.; Lebrilla, C. B.; Brown, P. H.; Plant Physiol. 1997, 113, 649 . 


\section{USO DA TÉCNICA HRICP-MS NA AVALIAÇÃO DOS ISÓTOPOS DE BORO EM EUCALIPTO}

Edson Marcio Mattiello*, Hugo Alberto Ruiz e Ivo Ribeiro da Silva

Departamento de Solos, Universidade Federal de Viçosa, 36570-000 Viçosa - MG, Brasil Jorge Eduardo de Souza Sarkis

Instituto de Pesquisas Energética e Nucleares, 05580-000 São Paulo - SP, Brasil

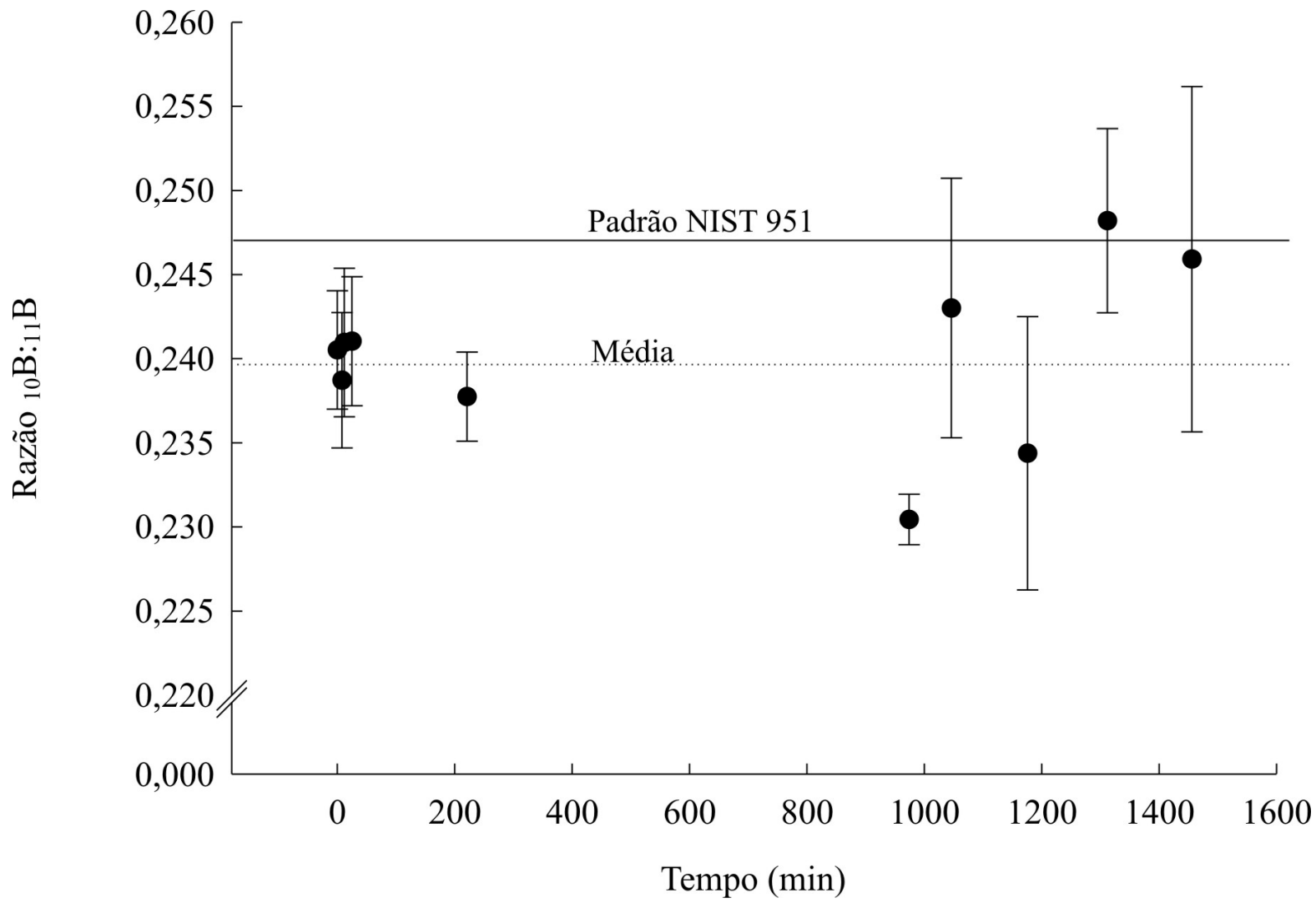

Figura 1S. Razão isotópica de B, considerando o tempo de duração das séries analíticas 